\title{
Effects of length, tonal structure, and contour in the recognition of tone series
}

\author{
W. L. M. CROONEN \\ Institute for Perception Research, Eindhoven, The Netherlands
}

\begin{abstract}
Four experiments on recognition of tone series are reported. The first experiment tested the accuracy of recognition in relation to length, contour complexity, and tonal structure of the series. Series comprised (1) 7 or 10 tones, (2) either a strong or a weak tonal structure, depending on the temporal ordering of the tones, and (3) few or many contour reversals. The second experiment used 7-tone series having either a strong or a weak tonal structure, depending on the mode (Ionian or Phrygian) in which the series was presented. Both experiments employed a samedifferent task in which a standard series was compared with either an exact or an inexact transposition, the latter type having one incorrectly transposed tone (mostly nondiatonic in Experiment 1 and always diatonic in Experiment 2). These experiments showed that (1) 7-tone series were better recognized than were 10-tone series, (2) series with a strong tonal structure were better recognized than were series with a weak tonal structure, and (3) contour complexity did not influence the responses. Two control experiments, using mistuned tone series, showed that the outcomes of Experiments 1 and 2 could not be attributed to nonmusical artifacts of the stimulus set.
\end{abstract}

Research into the field of memory for tone series has shown that a number of melodic features are of major importance in series recognition or recall. The contour of a series, which can be defined as the pattern of ascending and descending intervals, appears to be a basic memory factor in many studies (e.g., Dowling, 1978; Dowling \& Fujitani, 1971). Both musically trained and untrained subjects can easily extract this feature from a novel series and use it in a recognition task.

A second factor of importance is the "musical structure" of the series. This general term relates to the very tones that are used in the series and their ordering. The musical structure of a series has been the subject of many experimental studies, and it has been defined in several ways. A relatively simple way of defining it is to specify from which scale the tones of a series have been taken (e.g., diatonic series consisting of tones taken from the diatonic scale). An interesting outcome of some studies using this definition of musical structure is that diatonic series are generally better recognized than nondiatonic series (e.g. , Dowling, 1978; Francès, 1958). Another way of defining musical structure is to relate it to musictheoretical concepts, such as "cadential structure." The large number of studies conducted on the role of this kind of musical structure in recognition suggest that series con-

The author would like to thank Adrian Houtsma, Annabel Cohen, Helen Brown, Bea de Gelder, and Shari Campbell for their useful comments. The research was supported by a joint research program of Eindhoven University of Technology and Tilburg University. Correspondence should be addressed to W.L.M. Croonen, Institute for Perception Research, P.O. Box 513, 5600 MB Eindhoven, The Netherlands (e-mail: croonen@prl.philips.nl).

-Accepted by previous editor, Charles W. Eriksen structed along simple music-theoretical rules are better recognized (e.g., Cuddy \& Lyons, 1981; Dewar, Cuddy, \& Mewhort, 1977). Finally, Deutsch and Feroe (1981) proposed that tone series are retained as hierarchies of melodic-interval structures, each associated with a given pitch alphabet. According to Deutsch (1980), listeners perceive hierarchical structures present in tone series and use these structures in recall. The degree to which a series can be recalled appears to depend on the degree to which it can be coded parsimoniously.

Another important characteristic in tone-series recognition is duration of the retention interval (RI; the time interval between two series, a standard and a comparison, to be compared in a recognition paradigm). Dowling and Harwood (1986) concluded that for recognition of novel tone series, contour is predominant in short-term memory with RIs up to $5 \mathrm{sec}$, and that the melodic-interval information is predominant with RIs of $30 \mathrm{sec}$ or longer. Croonen and Kop (1989) investigated recognition of tone series with short and long RIs, using series with a strong tonal structure (defined as stimuli consisting of a succession of triads in root position on Scale Steps I and VII successively, the series being concluded with a repetition of the tonic). Subjects made use of the melodic-interval information for both short and long RIs. Contour complexity, defined as the number of contour changes, was introduced as a separate variable. This variable did not influence the responses of the subjects. These authors concluded that melodic-interval information is easily abstracted from a series with a strong tonal structure (even within a few seconds), and that this factor is more important than contour complexity for recognition.

Dowling (1991) did a recognition experiment in which he varied the tonal strength of a series. In contrast to Croonen and Kop (1989) and Cuddy, Cohen, and Mewhort 
(1981), who defined tonally strong items as consisting of a succession of triads on important scale steps, Dowling's tonally strong stimuli started and ended on the tonic, constituted clearly tonal patterns in the key, and were "melodious" in the sense of using relatively small pitch intervals. In this way, they were similar to the diatonic stimuli used by Watkins (1985, Experiment 1). Using a continuous running memory task (Shepard \& Teghtsoonian, 1961), Dowling (1991) found that subjects made use of the melodic-interval information of tonally strong tone series with long, but not short, RIs and concluded that his earlier findings and those of Dewitt and Crowder (1986) were confirmed: Tonal strength has no influence when coupled with short retention times.

Though it seems that the results of Croonen and Kop (1989) and those of Dowling (1991) lead to conflicting conclusions, it must be stressed that a critical evaluation of both studies is complicated by important differences in experimental strategy. First, as also pointed out by Dowling, because Croonen and Kop used repeated standard series, it is likely that these authors did not study the same phenomenon as did Dowling, who used novel standard series only. In other words, the subjects participating in the experiments of Croonen and Kop might have memorized the standard series after a few trials, whereas the subjects participating in the experiment of Dowling were unable to develop a long-term standard. Second, Croonen and Kop used silent retention intervals in order to investigate whether untrained subjects could abstract interval information from a tone series. Dowling used a continuous running memory paradigm, in which standard series, transpositions and same-contour lures were presented continuously, with a delay of $11 \mathrm{sec}$ between the series; the task was to indicate, for each series, whether it had been presented before. In this paradigm, new standard series and/or comparisons could be presented within the RI between a standard series and its comparison, depending on the time delay between standard and comparison $(11,25,39$, or $53 \mathrm{sec})$. This is an important point, as the presentation of tone series within the retention interval is likely to cause interference, which influences recognition (Deutsch, 1970). In addition, it may be questioned whether Dowling's short- and long-term measures are comparable to those of Croonen and Kop. Dowling based his conclusions on a short-term memory measure (i.e., a retention time of $11 \mathrm{sec}$ ) with no interference and on three long-term memory measures (retention times of 25,39 , and $53 \mathrm{sec}$ ) with different amounts of interference $(1,2$, and 3 tone series, respectively).

Additional relevant information on the role of tonal strength in the recognition of tone series is to be found in a study by Cuddy et al. (1981, Experiment 2). This experiment used repeated standards that varied in degree of harmonic structure, ranging over five levels from strong to weak. A strong structure was defined as a succession of arpeggiated triads on important scale steps; this structure was weakened by altering (diatonically or non- diatonically) one or more tones. Cuddy et al. found a gradual decrease in the percentage of correct responses for these five levels, with the highest percentage correct for the series with the strongest harmonic structure. These data clearly support the view that the degree of harmonic structure of a tone series influences recognition. However, because a two-alternative forced-choice paradigm was used, in which a standard series was compared with an exact transposition (ET) and an inexact transposition (IT) within each trial, this study gives no clear answer to the question of whether this is true for both short and long retention times. The degree of harmonic structure was varied through altering one or more tones of a series with a strong structure. The purpose of the alteration(s) (either diatonic or nondiatonic) was to weaken the harmonic structure of the series. A consequence of the alteration of tones within a strong series was that tone series at four of the five levels of harmonic structure contained, in fact, different tone sets. Because the strength of the harmonic structure of the standard series was varied through altering their pitch content, these two variables were confounded. Consequently, the interpretation of the results of Cuddy et al. (1981, Experiment 2) is somewhat complicated. To further investigate the role of tonal strength in recognition of tone series, the present experiments used an experimental strategy with repeated standards and a silent short-term RI.

\section{EXPERIMENT 1}

A recognition experiment was performed to test whether subjects having no formal musical training are able to abstract melodic-interval information from a diatonic series with a strong tonal structure better than from a series with a weak structure. In contrast to some of the standard series used by Cuddy et al. (1981, Experiment 2), both strong and weak series contained the same diatonic tone set, the individual tones being presented in a different order. To investigate how the tonal/harmonic strength of the series relates to the length of the series, series consisting of either 7 or 10 tones were used. Finally, contour complexity was introduced as a separate variable. As indicated above, there are contrasting views about the role of contour complexity in the recognition of tone series. Some authors have shown that a complex contour (i.e., a contour with many reversals) complicates the recognition of series (e.g., Dowling \& Harwood, 1986). Croonen and Kop (1989) concluded that contour complexity does not influence recognition, provided that the series has a strong tonal structure. Contour complexity was introduced in the present experiment as a separate variable to investigate whether the results of Croonen and Kop (1989) concerning contour complexity could be confirmed and to further investigate the role of contour complexity in tone series with a weak tonal structure.

In the present experiment, a standard series was to be compared with either an ET or an IT, the latter having 
one incorrectly transposed tone that was mostly nondiatonically related to the key of the series. A yes/no task for identification of ETs was used.

\section{Method}

Subjects. Thirty subjects participated in the experiment (12 men, 18 women), ranging in age from 18 to 34 years. Some subjects played one or more instruments; however, none had undergone longterm formal performance training, nor had any received ear training. The subjects were tested individually and were paid for their participation. None of the subjects had participated in earlier experiments on tone-series recognition.

Apparatus. Tone series were compiled using sinusoids generated by a microVAX II computer and a DSC 200 D/A converter, sampled at $10 \mathrm{kHz}$ and lowpass filtered at $4.8 \mathrm{kHz}$. The series were recorded by means of a DAT recorder (Philips DAT 850), and played to the subjects through headphones (AKG 100) at a comfortable sound level. The experiment was performed in a soundinsulated room adjacent to the experimenter's room.

Stimuli. Eight standard series were compiled, each consisting of either 7 or 10 tones, a strong or a weak tonal structure, and few or many contour reversals. The standard series consisted of tones from the $\mathrm{C}$ major diatonic scale. The series are shown in Figure 1.

Standard Series 1 and 2 were taken from Cuddy et al. (1981, Experiment 2). These authors show that there are two possible chord
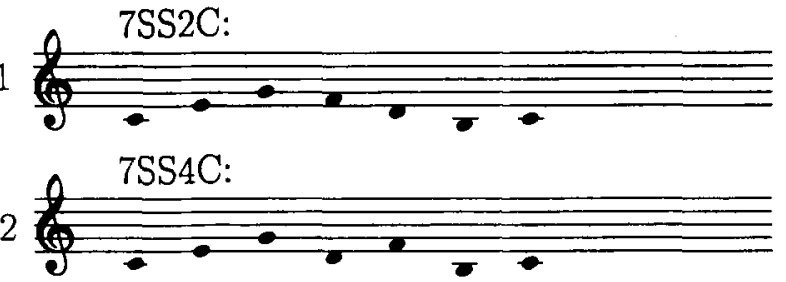

7WS2C:

3

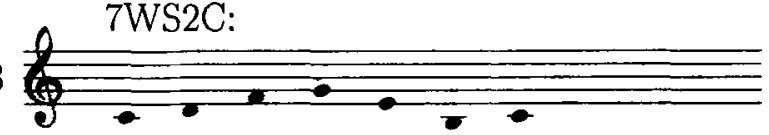

7WS4C:

4

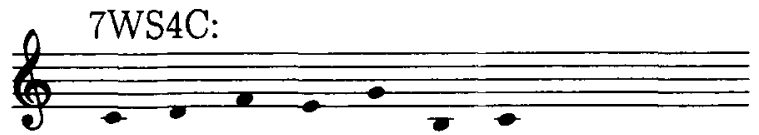

10SS3C:

5

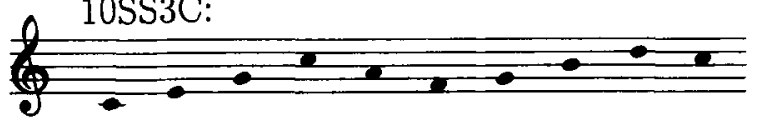

10SS5C:

6

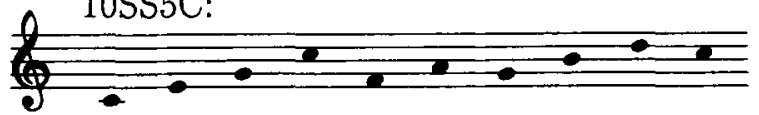

10WS3C:

7

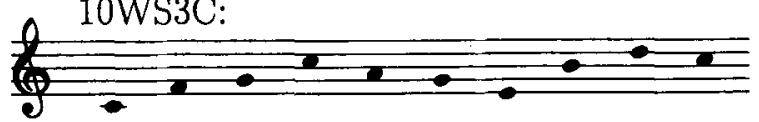

10WS5C:

8

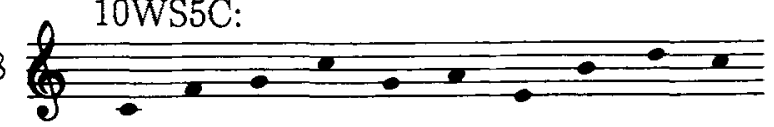

Figure 1. Standard series used in Experiment 1. The code indicated above each series shows (1) the number of tones ( 7 or 10 tones), (2) the structure of the series (SS = strong tonal structure, WS = weak tonal structure), and (3) the number of contour changes (few [ 2 or 3] or many [ 4 or 5$]$ ]. progression analyses for Series 1 (either a $I-v i i^{0}-I$ or a $I-V_{7}-I$ progression within the key of $\mathrm{C}$ major) and that these two chord progressions are closely related. The authors also explain in detail why the use of Series 1 as a prototypical series in a rating task should lead to the highest rating of perceived musical structure (Cuddy et al., 1981, Experiment 1); indeed, they found that listeners, regardless of their musical backgrounds, rated this series highest in tonal structure (Cuddy et al., 1981, Experiments 1 and 3). For these reasons, this series was also used in the present experiment as a prototype of a series with a strong tonal structure. Though Standard Series 2 has a complex contour, it has the same underlying chord progression as Series 1 , since music analysis is more concerned with sequential relations between groups of notes than within groups of notes (Cuddy et al., 1981, p. 871). Both Series 1 and 2 are tonally strong, because they are compiled according to highly specific music-theoretical rules. In order to compile a tonally weak series, one of these rules was violated. The tonally weak Series 3 and 4 contained the same diatonic tone set and the same number of contour changes as the tonally strong Series 1 and 2 , respectively, but the tones were presented in a different order, resulting in a violation of the triadic chord structure of the series. In other words, there was no strong $\left(I-V_{7}-I\right.$ or $\left.I-v i i^{0}-I\right)$ underlying chord progression in Series 3 and 4.

Series 5-8, consisting of 10 tones, were compiled along the same lines as Series 1-4. First, a prototypical tonally strong series was compiled (Series 5) using tones of major triads in root position on scale steps $I, I V$, and $V$ within the key of $C$ major; the series was concluded with a repetition of the tonic. Krumhansl, Bharucha, and Kessler (1982) have shown that triads on scale steps $I, V$, and $I V$ are closely related. They have stated that a harmonic progression involving these chords gives what may be the strongest possible establishment of a major key. As in Series 1-4, chords were presented in an arpeggiated fashion. Series 6-8 contained the same tone set as Series 5 , but differed from this series in the following respects. Series 6 had the same underlying chord progression as Series 5 , but had a more complex contour. Series 7 had the same number of contour reversals as Series 5 , but had no strong underlying chord progression, as the temporal order of the tones was changed. Finally, Series 8 had a more complex contour than Series 5 and no strong underlying chord progression.

The eight standard series can be described as follows: (1) 7 tones, strong tonal structure and 2 contour changes (7SS2C); (2) 7 tones, strong tonal structure and 4 contour changes (7SS4C); (3) 7 tones, weak tonal structure and 2 contour changes (7WS2C); (4) 7 tones, weak tonal structure and 4 contour changes (7WS4C); (5) 10 tones, strong tonal structure and 3 contour changes (10SS3C); (6) 10 tones, strong tonal structure and 5 contour changes (10SS5C); (7) 10 tones, weak tonal structure and 3 contour changes (10WS5C); and (8) 10 tones, weak tonal structure and 5 contour changes (10WS5C).

The comparison series was either an ET or an IT of the standard series to one of the following keys: F maj., Ab maj., B maj., C maj., or $E b$ maj., which are all (reasonably) far removed from the key of $\mathrm{C}$ major on the circle of fifths. An IT was a transposition with one incorrectly transposed note; this note was at a different position for each of the five ITs, and the first and the last note was always transposed correctly. In line with the experiment of Cuddy et al. (1981, Experiment 2), but contrary to that of Dowling (1991), the incorrectly transposed note was a semitone below the correctly transposed note. For this reason, this tone was nondiatonically related to the key of the transposition in $80 \%$ of the trials. The incorrectly transposed tone was diatonically related for the notes occurring on the fourth scale degree, as this note was changed into the diatonic third degree, by lowering it a half tone. The contour of the ITs remained the same as that of the standard. The tones of the series had a total duration of $300 \mathrm{msec}$ (with a rise and fall time of $20 \mathrm{msec}$ ), and the pauses between the tones were $50 \mathrm{msec}$. There was a 3-sec silent RI between standard and comparison.

Procedure. It was explained to the subjects that, for each trial, two tone series were to be compared, the second being either an 
ET or an IT of the first, where ITs contained only one incorrectly transposed note. The subjects were informed that half of the trials had an ET comparison and the other half had an IT comparison. The experiment started with six trials in which a well-known tune was to be compared with either an ET or an IT. For these six trials, the experimenter gave correct-answer feedback. If there were no more questions after the introductory part of the experiment, the first block of trials was presented.

The whole experiment consisted of 80 trials (5 ETs and 5 ITs for each of the 8 standards) divided into two blocks of 40 trials, one containing the 7-tone series and the other containing the 10tone series. Fifteen subjects started with the 7-tone series block, and the remaining 15 subjects started with the 10-tone series block. The order of presentation of trials within each block was randomized and was the same for each subject. Each trial started with a short warning tone $(1000 \mathrm{~Hz}, 10 \mathrm{msec})$, followed by a $2-\mathrm{sec}$ pause. The standard series was then presented, followed by a silent RI of $3 \mathrm{sec}$, after which the comparison was presented. A 7-sec interval followed in which the subject was to respond by marking one of two alternatives on a response form: same for an ET comparison (having the same set of intervals as the standard) and different for an IT comparison (having two intervals different from the standard). A separate response form was used for each block. There was a pause of about $5 \mathrm{~min}$ between the blocks, in which a short inventory was completed on the musical background of the subject.

\section{Results}

Sensitivity and bias. Using signal detection theory (Green \& Swets, 1966; Swets, 1973), the responses were transformed into proportions of hits (correct recognition of a transposition) and false alarms (incorrect recognition of a transposition). Macmillan, Kaplan, and Creelman (1977) developed an analysis of same-different data through an extension of signal detection theory, and Kaplan, Macmillan, and Creelman (1978) published ta-

Table 1

$d^{\prime}$ and $\beta$ Measures in Experiment 1

\begin{tabular}{ccc}
\hline Factor & $d^{\prime}$ & $\beta$ \\
\hline Length & & \\
7 tones & 2.16 & -.11 \\
10 tones & 1.62 & .04 \\
Structure & & .23 \\
SS & 2.16 & -.06 \\
WS & 1.65 & -.04 \\
Contour & & -.12 \\
Simple & 1.84 & \\
Complex & 1.94 &
\end{tabular}

Note $-N=30$.

Table 2

Mean Estimated Areas Under MOC in Experiment 1

\begin{tabular}{lcccccc}
\hline & \multicolumn{2}{c}{ Structure } & & \multicolumn{2}{c}{ Contour } & \\
\cline { 2 - 3 } \cline { 5 - 6 } Factor & SS & WS & & Simple & Complex & $M$ \\
\hline Length & & & & & \\
7 tones & 82.10 & 69.02 & & 72.42 & 78.69 & 75.56 \\
10 tones & 69.26 & 65.72 & 68.47 & 66.51 & 67.49 \\
Structure & & & & & \\
$\quad$ SS & & & 78.27 & 73.08 & 75.68 \\
$\quad$ WS & & & 62.62 & 72.12 & 67.37 \\
Contour & & & & & \\
$\quad$ Simple & & & & & 70.44 \\
Complex & & & & & 72.60 \\
\hline
\end{tabular}

Note $-N=30$.

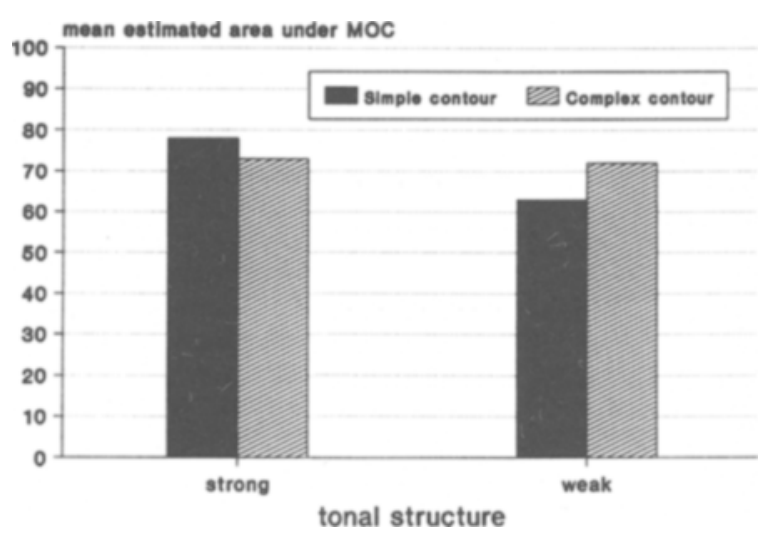

Figure 2. Mean estimated areas under the MOC for two levels of tonal strength (strong and weak) and two levels of contour complexity (simple or complex).

bles for analysis of this kind of data. Group average hit and false-alarm rates were used to derive the $d$ ' measures shown in Table 1 . In Table 1 , it can be seen that all $d^{\prime}$ scores are well above threshold level, as defined by $d^{\prime}>$ 1.0 (Green \& Swets, 1966). In general, the discrimination of short series and of series with a strong tonal structure is better than the discrimination of long series and of series with a weak tonal structure. There was, on average, a slight negative bias.

The hit and false-alarm proportions were used to calculate the estimated area under the memory operating characteristic (MOC; Banks, 1970; Norman, 1964; Norman \& Wickelgren, 1965; Pollack \& Norman, 1964; Pollack, Norman, \& Galanter, 1964). It has been shown that the estimated area under the operating curve is a way of assessing performance in recognition experiments in terms of an equivalent forced-choice score if there is no response bias (Pollack \& Norman, 1964). Mean estimated area scores are presented in Table 2 . The estimated area scores were normalized using an arcsine transformation rule (McNicol, 1971).

Analysis of variance. An analysis of variance (ANOVA) was performed on the normalized estimated area scores, with the following factors: (1) subjects (random factor), (2) length of the series (two levels: 7 and 10 tones), (3) degree of tonal structure (two levels: strong tonal structure or weak tonal structure), and (4) contour complexity (two levels: 2 [for 10-tone series, 3] changes or 4 [for 10-tone series, 5] changes).

The ANOVA yielded main effects of length $[F(1,29)=$ $20.75, p<.001]$ and structure $[F(1,29)=12.06, p<$ $.01]$. There was no effect of contour $[F(1,29)=0.36$, $p=.55]$. Furthermore, there were significant interactions between length and structure $[F(1,29)=5.58, p<.05]$ and, as shown in Figure 2, between structure and contour $[F(1,29)=11.80, p<.01]$.

In order to determine which pairs were significantly different, post hoc Scheffé pairwise analyses (Scheffé, 1953) were conducted. For the interaction between length and structure, results showed that 7 -tone series with a strong 
tonal structure were better recognized than any other combination. For the interaction between structure and contour, results showed that series with a simple contour and a strong tonal structure were significantly better recognized than series with a simple contour and a weak tonal structure.

\section{Discussion}

The present data show that the degree of tonal structure is an important factor in tone-series recognition with short retention times. Within $3 \mathrm{sec}$, the subjects did abstract the melodic intervals from a series with a strong tonal structure significantly better than from a series with a weak structure, both types of series consisting of the same set of tones. This conclusion is in line with the results of Croonen and Kop (1989), who also found that untrained subjects recognize the melodic-interval structure of a series with a strong tonal structure better than that of a series with a weak tonal structure. This finding is also in line with the conclusion of Cuddy et al. (1981), concerning the role of harmonic structure in recognition of tone series mentioned above.

The importance of tonal structure in tone-series recognition is also stressed by Dewar et al. (1977), who found that, for trained and untrained subjects, tones are better recognized when presented within a tonal context than when presented in isolation. Further support for the importance of tonal structure is given by Beal (1985), who found that musically trained subjects use the harmonic structure of chords in deciding whether or not two successively presented chords are the same, whereas musically untrained subjects do not. The present experiment shows that untrained subjects discriminate ETs and ITs of a tone series with a strong tonal structure significantly better than they discriminate ETs and ITs of series having a weak structure. It can be concluded that untrained subjects also use this type of information in deciding whether or not two series have the same set of intervals.

A second important factor appears to be the length of the series. Seven-tone series are better recognized than are 10-tone series. Length and structure interact in such a way that short series with a strong structure are far better recognized than are any other combination of these characteristics.

The present results are not in line with the conclusion of Cuddy et al. (1981) concerning contour, since it was not found that contour complexity influences recognition. This finding is in line, however, with the conclusion of Croonen and Kop (1989) concerning the role of contour complexity mentioned above. This issue will be further discussed below.

In summary, it can be concluded that, in an experimental paradigm with repeated standards and a silent RI of a few seconds, both tonal structure and length of the series are important determinants for series recognition. The main conclusion is that, for short tone series (the 7-tone series used in the present experiment), the degree of tonal structure is a major determinant of series recognition. Fur- thermore, it was found that series with many contour reversals are recognized as well as series with few contour reversals.

\section{EXPERIMENT 2}

The results of Experiment 1 lead to the conclusion that a strongly tonal structure is of importance in recognizing tone series with short retention times. However, an alternative explanation for the findings of Experiment 1 may arise from the fact that the ITs had only one incorrectly transposed tone, which was, in $80 \%$ of the trials, nondiatonically related to the key of the comparison. In this way, the task employed in Experiment 1 consisted largely of a comparison between a diatonic and a nondiatonic series for the IT trials and between two diatonic series for the ET trials. The subjects might have used diatonicity or nondiatonicity of the comparison series as a cue for same versus different trials, which may have simplified the task; Dowling (1978) has shown that, in general, diatonic series could easily be discriminated from nondiatonic series.

There may be yet another explanation for the diminished recognition of series of which the tonal strength has been weakened through reordering the tones. The weak series of Experiment 1 consisted of small and large melodic intervals, whereas the strong series generally had a more regular melodic-interval structure. As shown by Boltz and Jones (1986), an irregular melodic-interval structure is not recognized as well as is a regular structure. These authors attributed this result to the fact that unexpectedly occurring large or small melodic intervals get a melodic "accent"; series having relatively many irregularly occurring accents are more difficult to retain than are series having regularly occurring accents. The reordering of tones of a strong series may have influenced, next to the tonal strength, tonic specificity and ambiguity (Brown \& Butler, 1981).

As with Experiment 1, a second experiment was performed primarily to investigate whether tonal structure, as defined by Croonen and Kop (1989) and Cuddy et al. (1981), is of importance in tone-series recognition with short retention times. The aim of Experiment 2 was to investigate whether the conclusions of Experiment 1 can be generalized to recognition of strongly and/or weakly tonal diatonic tone series. ETs and ITs were used, the latter having one incorrectly transposed tone, which was (contrary to Experiment 1) diatonically related to the key of the transposition. Furthermore, tonally strong series were transformed into tonally weak series by presenting them in the Phrygian mode (Dowling, 1991). This way of weakening the tonal strength of a series does not create a more irregular intervallic structure for the weak series relative to that for the strong series; consequently, there is no different accent structure for the weak series relative to that for the strong series. The task was again a yes/no identification of correct transpositions, using a silent retention interval. 


\section{Method}

Subjects. Twenty subjects participated in the experiment (16 men, 4 women), ranging in age from 21 to 35 years. All subjects were interested in music. Some of them played one or more instruments; however, none had undergone long-term professional training in playing an instrument, nor had any received ear training. The subjects were tested individually and were paid for their participation. None of the subjects had participated in Experiment 1.

Apparatus. Tone series were compiled using sinusoids generated alternatingly by two Philips PM 5190 LF synthesizers, controlled by a microVAX II computer. The series were presented through Telephonics TDH 49 headphones at a comfortable listening level. The experiment was performed in a sound-insulated room adjacent to the experimenter's room.

Stimuli. Eight standard series were compiled: four tonally strong series (SS1-4), and four tonally weak series (WS1-4). The four tonally strong series were compiled along the criteria used in Experiment 1 , with an underlying $I-v i i^{0}-I / I-V_{7}-I$ chord sequence in the key of $\mathrm{C}$ major. The tonally weak series consisted of the tonally strong series presented in the Phrygian mode. Standard series are presented in Figure 3.

For each of these standard series, 10 comparisons were compiled: 5 ETs and 5 ITs. The ETs consisted of transpositions of the standard to the following keys: $F \$$ maj., Ab maj., B maj., C maj., or Eb maj. The ITs also consisted of transpositions of the standard
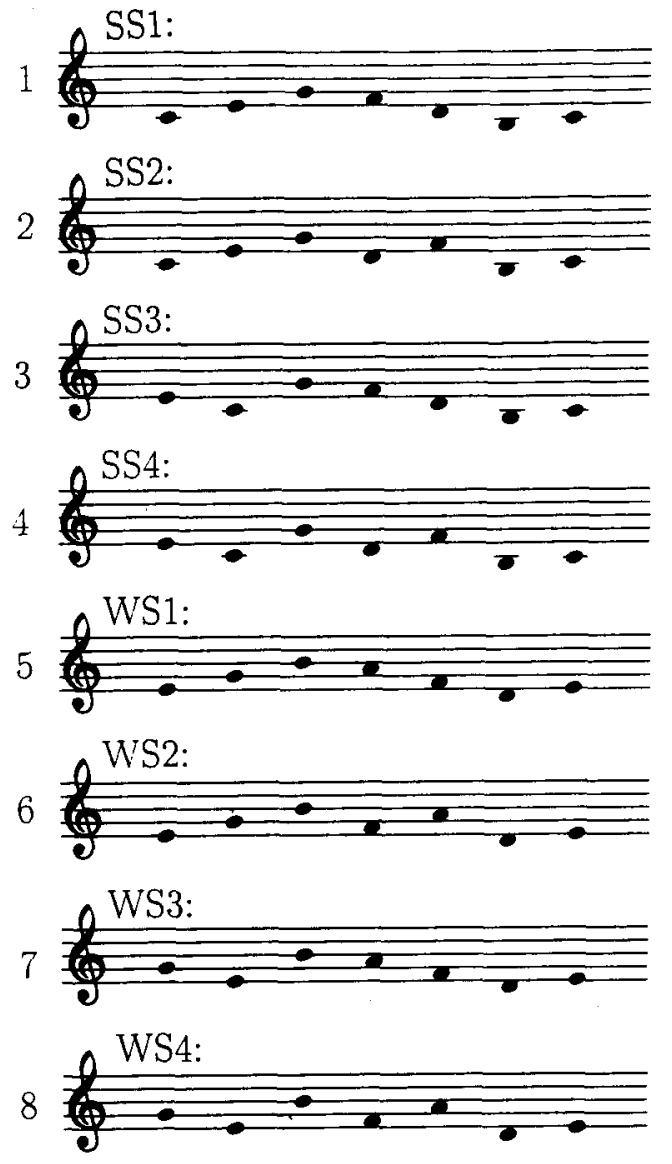

Figure 3. Standard series used in Experiment 2. Tone series labeled SS1-SS4 had a "strong" tonal structure, consisting of a triadic tonic-dominant-tonic progression. Series labeled WS1-WS4 had a "weak" structure, consisting of the strong series presented in the Phrygian mode.
Table 3

$d^{\prime}$ and $\beta$ Measures in Experiment 2

\begin{tabular}{ccc}
\multicolumn{3}{c}{$\boldsymbol{d}^{\prime}$ and $\boldsymbol{\beta}$ Measures in Experiment 2} \\
\hline Factor & $d^{\prime}$ & $\beta$ \\
\hline Structure & & \\
SS & 3.49 & -0.09 \\
WS & 2.35 & -0.12 \\
\hline
\end{tabular}

Note $-N=20$.

to the above-mentioned keys, but in each IT one note was transposed incorrectly. This incorrectly transposed note was one diatonic step either below or above the correctly transposed note, depending on the contour of the comparison, which was to be the same as that of the standard. For each of the five ITs, the incorrectly transposed note was at a different serial position, and the first and the last tone of the standard was always transposed correctly. The tones had a total duration of $300 \mathrm{msec}$ (with rise and fall time of $20 \mathrm{msec}$ ), and the pauses between the tones were $50 \mathrm{msec}$.

Procedure. The subjects were familiarized with the experimental setting in the same way as was done in Experiment 1 . The 80 trials were presented in random order; the particular randomization was different for each subject. Each trial started with a short warning tone $(1000 \mathrm{~Hz}, 10 \mathrm{msec})$, followed by a 2 -sec pause. The standard series was then presented, followed by a silent RI of $3 \mathrm{sec}$, after which the comparison was presented. After the presentation of the comparison, the subjects responded by pressing one of two buttons: same for an ET comparison (having the same set of intervals as the standard) and different for an IT comparison (having two different intervals from the standard). Response time was unlimited. The subjects received no feedback concerning the correctness of the response.

\section{Results}

Sensitivity and bias. The responses were transformed into proportions of hits (correct recognition of a transposition) and false alarms (incorrect recognition of a transposition). Group average $d^{\prime}$ measures (Kaplan et al., 1978; Macmillan et al., 1977) are shown in Table 3. In Table 3, it can be seen that both $d^{\prime}$ 's are well above threshold level, as defined by $d^{\prime}>1.0$ (Green \& Swets, 1966). In general, the discrimination of series presented in the Ionian mode was worse than the discrimination of series presented in the Phrygian mode. There was, on average, a slight negative bias.

The hit and false-alarm proportions were used to calculate the estimated area under the MOC. Mean estimated area scores for SS and WS were 85.92 and 79.92, respectively. The estimated area scores were normalized using an arcsine transformation rule (McNicol, 1971).

Analysis of variance. An ANOVA was performed on the normalized estimated area scores, with the following factors: (1) subjects (random factor), and (2) degree of tonal structure (two levels: strong tonal structure or weak tonal structure). The ANOVA yielded a main effect of structure $[F(1,19)=12.75, p<.01]$.

\section{Discussion}

In line with the results of Experiment 1, the results of Experiment 2 show that subjects can discriminate between ETs and ITs of a tone series with a strongly tonal (triadic) structure using a silent RI of $3 \mathrm{sec}$. As discussed by Dowling (1991), discrimination performance decreases significantly when a strongly tonal series is presented in 
the Phrygian mode, probably because the tonal strength of the series has decreased. In Experiment 2, all standards and comparisons consisted of diatonically related tones: unlike in Experiment 1, the results of Experiment 2 cannot be explained as a comparison between a diatonic and a nondiatonic series (IT trials) or two diatonic series (ET trials).

The results of Experiments 1 and 2 show that untrained listeners discriminate ETs and ITs of a series better when the ITs have a diatonically altered tone (Experiment 2) than when they have a nondiatonically altered tone (Experiment 1). This is contrary to what was to be expected from earlier reports (e.g., Dowling, 1978), which suggest that diatonic series are more easily discriminated from nondiatonic series than from diatonic series. This finding is not easily explained. However, the diatonic ITs generally contain an altered tone one scale step below the correctly transposed tone, whereas the nondiatonic ITs contain an alteration a half scale step away from the correctly transposed tone. In other words, in the present experiment, because the diatonic alterations are, in general, larger than the nondiatonic alterations, it is possible that our subjects more easily discriminated between series having relatively large alterations than between series having relatively small alterations.

The results of both experiments show clearly that subjects with no long-term formal musical training can abstract melodic-interval information from a series with strong tonal structure much better than from a series with a weak structure, at least for a retention time of $3 \mathrm{sec}$. In both experiments, a strong structure was defined in a music-theoretical way: a cadential succession of arpeggiated chords in a tonic-dominant-tonic relation. The decreased performance for series with a weak structure appears to be independent of the way in which the weak series was compiled, either through rearranging the (triadic) temporal order of the tones of a strong series or through presenting a strong series in the clearly less tonal Phrygian mode. The present results confirm the results of Croonen and Kop (1989) and Cuddy et al. (1981) concerning the major role of the tonal strength of a series for recognition.

Two control experiments were performed in order to check for adequacy of the manipulation of the tonal strength variable in Experiments 1 and 2 . It is possible that the constraints associated with manipulating this variable in Experiments 1 and 2 led to one or more nonmusical artifacts (e.g., contours that are, as such, easier to remember than others). In addition, as noted above, the better overall discrimination performance in Experiment 2 relative to that in Experiment 1 might be attributed to the fact that the sizes of the alterations were generally larger for the diatonic (Experiment 2) than for the nondiatonic (Experiment 1) alterations.

A useful way of controlling nonmusical artifacts is provided by Watkins (1985), who used series of tones with either tuned or mistuned melodic intervals to separate effects due to music-perceptual knowledge from more gen- eral perceptual factors. "Tuned" tone series contain note frequencies based on the 12-tone equal temperamentthat is, the octave is divided into 12 equal semitones (the smallest interval) by the twelvefold division of the 2:1 ratio of the octave. For "mistuned" tone series, the smallest interval is increased or decreased in size by a constant (log) amount. In this way, the octave is divided into more or fewer than 12 intervals. Tonality effects involving specific music-perceptual knowledge, as found in Experiments 1 and 2 , should be destroyed by the use of mistuned tone series, whereas nonmusical artifacts should not.

\section{EXPERIMENT 3}

Mistuning as used by Watkins (1985) and in the present Experiments 3 and 4 can be explained as follows. The constant frequency ratio that relates the notes of adjacent semitones (called quantal intervals by Watkins, 1985) within equal-temperament tuning, can be described with the formula

$$
f / f_{0}=2^{1 / 12},
$$

where $f_{0}$ is the frequency of the reference note and $f$ is the frequency of a note a semitone above this reference. As Watkins explains, it is possible to define an integer for a given note that represents the number of semitones between the note frequency and a reference note frequency, by using the formula

$$
f / f_{0}=2^{p / 12}
$$

where $p$ represents the number of semitones between note $f$ and reference $f_{0}$. The frequency ratio of the quantal interval can be varied by a constant amount with the introduction of a scaling factor $c$ :

$$
f / f_{0}=2^{\mathrm{cp} / 12} .
$$

For the equal-temperament tuning, this scaling factor is 1.0 , resulting in a twelvefold division of the octave. Mistuning can be done in two ways: the quantal interval can be contracted by using a scaling factor $c<1.0$, or it can be expanded by using a scaling factor $c>1.0$.

\section{Method}

Subjects. Twenty-five subjects participated in the experiment (20 men, 5 women), ranging in age from 21 to 43 years. Some subjects played one or more instruments; however, none had undergone long-term professional training in playing an instrument, nor had any received ear training. The subjects were tested individually.

Apparatus and Stimuli. The apparatus was the same as that used in Experiment 2. The stimuli were the tone series used in Experiment 1, presented in a mistuned fashion. For this, the quantal interval was scaled by a factor of 0.7 , resulting in a quantal interval size of 70 cents, where 1 cent is one hundredth of a semitone.

Procedure. The subjects were familiarized with the experimental setting in the same way as was done in Experiment 1, but were told that the series would sound quite "nonmusical." Contrary to Experiment 1, the 80 trials were presented in a different random order for each subject. Each trial started with a short warning tone $(1000 \mathrm{~Hz}, 10 \mathrm{msec}$ ), followed by a $2-\mathrm{sec}$ pause. The standard se- 
ries was then presented, followed by a silent RI of $3 \mathrm{sec}$, after which the comparison was presented. After the presentation of the comparison, the subjects responded by pressing one of two buttons: same for an ET comparison (having the same set of intervals as the standard) and different for an IT comparison (having two intervals different from the standard). Response time was unlimited. The subjects received no feedback concerning the correctness of the response.

\section{Results and Discussion}

Sensitivity and bias. The responses were transformed into proportions of hits (correct recognition of a transposition) and false alarms (incorrect recognition of a transposition). The hit and false-alarm proportions were used to calculate the estimated area under the MOC. Mean estimated area scores are presented in Table 4 . The estimated area scores were normalized using an arcsine transformation rule (McNicol, 1971).

Group average $d^{\prime}$ measures (Kaplan et al., 1978; Macmillan et al., 1977) are shown in Table 5. In Table 5, it can be seen that all $d^{\prime}$ scores are below threshold level, as defined by $d^{\prime}>1.0$ (Green \& Swets, 1966). There was, on average, a negative bias, which resulted in an underestimate of the area under the MOC and, consequently, in an underestimate of the percentage of correct responses. However, taking into account that the negative bias was (nearly) equal for all conditions, the degree of underestimation of the recognition performance was the same for all conditions. Consequently, the outcome of the ANOVA cannot be attributed to differential estimation of recognition performance for different conditions.

Table 4

Mean Estimated Areas Under MOC in Experiment 3

\begin{tabular}{|c|c|c|c|c|c|}
\hline \multirow[b]{2}{*}{ Factor } & \multicolumn{2}{|c|}{ Structure } & \multicolumn{2}{|c|}{ Contour } & \multirow[b]{2}{*}{$M$} \\
\hline & SS & WS & Simple & Complex & \\
\hline \multicolumn{6}{|l|}{ Length } \\
\hline 7 tones & 54.94 & 59.88 & 58.00 & 56.82 & 57.41 \\
\hline 10 tones & 54.18 & 50.73 & 51.69 & 53.23 & 52.46 \\
\hline \multicolumn{6}{|l|}{ Structure } \\
\hline SS & & & 51.71 & 57.41 & 54.56 \\
\hline WS & & & 57.97 & 52.64 & 55.30 \\
\hline \multicolumn{6}{|l|}{ Contour } \\
\hline Simple & & & & & 54.84 \\
\hline Complex & & & & & 55.02 \\
\hline
\end{tabular}

Note $-N=25$.

Table 5

$d^{\prime}$ and $\beta$ Measures in Experiment 3

\begin{tabular}{|c|c|c|}
\hline Factor & $d^{\prime}$ & $\beta$ \\
\hline \multicolumn{3}{|l|}{ Length } \\
\hline 7 tones & .90 & -.21 \\
\hline 10 tones & .51 & -.19 \\
\hline \multicolumn{3}{|l|}{ Structure } \\
\hline SS & .79 & -.19 \\
\hline WS & .79 & -.23 \\
\hline \multicolumn{3}{|l|}{ Contour } \\
\hline Simple & .79 & -.21 \\
\hline Complex & .66 & -.21 \\
\hline
\end{tabular}

Note $-N=25$.
Analysis of variance. An ANOVA was performed on the normalized estimated area scores, with the following factors: (1) subjects (random factor), (2) length (two levels: 7 tones or 10 tones), (3) degree of tonal structure (two levels: strong tonal structure or weak tonal structure), and (4) contour complexity (two levels: 2 [for 10tone series, 3] changes or 4 [for 10-tone series, 5] changes). The ANOVA yielded no significant main effects and no significant interactions. The $F$-ratios and corresponding significance levels for the main factors were: length, $F(1,24)=2.72, p=.11$; structure, $F(1,24)=$ $0.07, p=.79 ;$ and contour, $F(1,24)=0.00, p=.99$.

Both the outcome of the ANOVA and the sensitivity measures show that it was virtually impossible for this group of untrained subjects to discriminate between ITs and ETs of any type of standard series. Though 7-tone series were, in general, somewhat better recognized than were 10-tone series, none of the variables reaching significance in Experiment 1 reached significance in Experiment 3 . This outcome leads to the conclusion that the results of Experiment 1 cannot be attributed to nonmusical artifacts of the stimulus set.

\section{EXPERIMENT 4}

In order to investigate whether the results of Experiment 2 concerning the role of tonal strength in tone-series recognition can be attributed to nonmusical artifacts, a control experiment was performed. Mistuning was used as a control factor for reasons outlined above and was introduced in the way described in Experiment 3.

\section{Method}

Subjects. Twenty subjects participated in the experiment (16 men, 4 women), ranging in age from 21 to 36 years. Some subjects played one or more instruments; however, none had undergone long-term professional training in playing an instrument, nor had any received ear training. The subjects were tested individually.

Apparatus and Stimuli. The apparatus was the same as that used in Experiment 2. The stimuli were the tones series used in Experiment 2, presented in a mistuned fashion (as described in the introduction to Experiment 3 ). The scaling factor by which the quantal interval was contracted was 0.7 .

Procedure. The procedure was the same as that used in Experiment 3.

\section{Results and Discussion}

Sensitivity and bias. The responses were transformed into proportions of hits (correct recognition of a transposition) and false alarms (incorrect recognition of a transposition). The hit and false-alarm proportions were used to calculate the estimated area under the MOC. Mean estimated area scores for SS and WS were 70.81 and 65.93, respectively. The estimated area scores were normalized using an arcsine transformation rule (McNicol, 1971).

Group average $d^{\prime}$ measures (Kaplan et al., 1978; Macmillan et al., 1977) are shown in Table 6. In Table 6, it can be seen that $d^{\prime}$ scores are above threshold level, as described by $d^{\prime}>1.0$ (Green \& Swets, 1966). Furthermore, it can be seen that there was, on average, a slight negative bias. 
Table 6

$d^{\prime}$ and $\beta$ Measures in Experiment 4

\begin{tabular}{ccc}
\hline Factor & $d^{\prime}$ & $\beta$ \\
\hline Structure & & \\
SS & 1.83 & -.09 \\
WS & 1.53 & -.06 \\
\hline
\end{tabular}

Note $-N=20$.

Analysis of variance. An ANOVA was performed on the normalized area scores, with the following factors: (1) subjects (random factor) and (2) degree of tonal structure (two levels: strong tonal structure or weak tonal structure). The ANOVA yielded no significant main effect. The $F$ ratio and corresponding significance level for structure was $F(1,19)=2.79, p=.11$.

As in Experiment 3, the results show that this group of subjects could not discriminate between ITs and ETs of the mistuned set of standard series. Though the recognition performance for strongly structured versus weakly structured series was generally better than the performance in Experiment 3, this variable did not reach significance. As in Experiment 2, it can be concluded that the effect of tonal strength as found in Experiment 2 cannot be attributed to nonmusical artifacts of the stimulus set.

\section{GENERAL DISCUSSION}

Experiment 1 showed that, using an experimental paradigm with repeated standards and silent short-term RIs, tonal strength and series length are major factors in recognition of tone series by listeners without formal musical training. Experiment 2 also showed that tonal strength is of major importance in series recognition and, in addition, that the effect cannot be attributed to discrimination of nondiatonic versus diatonic series, which was a possible alternative explanation for the outcome of Experiment 1. Experiments 3 and 4, using mistuned tone series, showed that the results of Experiments 1 and 2 could not be attributed to nonmusical artifacts of the stimulus set. The outcomes of the present experiments lead to the conclusion that, within the present experimental paradigm, musically untrained listeners use the melodic-interval information of a tonally strong series in deciding whether two series are exactly transposed or not. However, they apparently cannot use the melodic-interval information of a tonally weak series. This conclusion is consistent with that of Cuddy et al. (1981), concerning the role of harmonic structure in recognition of tone series. Furthermore, tonal strength appears especially useful for recognition of relatively short series: 7-tone series were recognized significantly better than were 10-tone series.

An additional result of Experiment 1 was that contour complexity has no influence on series recognition. Although this is not in line with previous conclusions (e.g., Cuddy et al., 1981), which state that series with many contour reversals are less well recognized than are series with few reversals, these results do support Croonen and Kop (1989). These authors concluded that subjects attend to the underlying tonal scheme instead of to the pattern of "ups and downs." This is still a plausible explanation of their results and those of the present experiments, but there is also a difference between the tasks employed by Cuddy et al. (1981) and Croonen and Kop (1989). The latter used a same-different task (as in the present experiments) with silent RIs. Cuddy et al. used a two-alternative forced-choice task in which a standard series was to be compared with two comparisons in each trial. Interference also plays a role in a two-alternative forced-choice task, as there are no silent RIs; the subject must listen to two comparisons and choose the correct item. The tones of the series presented in the first interval may very well have influenced the way the subjects listened to the series presented in the second interval. The difference between the outcome of the present experiments and those of Cuddy et al. (1981) is not explained by the difference in the experimental task employed; however, since it has been shown that specific auditory interference plays a part in auditory recognition, the difference between the tasks makes the two experiments only slightly comparable. Further research on this issue is needed.

As mentioned above, the results of Experiments 1 and 2 showed that untrained listeners discriminate better between ETs and ITs of a series, when the ITs have a diatonically altered tone (Experiment 2) instead of a nondiatonically altered tone (Experiment 1 ). The results of Experiments 3 and 4 show that there was also a better recognition performance for the mistuned stimulus set of Experiment 2 than for the mistuned stimulus set of Experiment 1. As suggested above, a plausible explanation for this difference in performance, one that arises in both equal-temperament and nonequal-temperament tuning, is that the alterations used in Experiment 2 were, in general, larger than the alterations used in Experiment 1, and the alterations in Experiment 4 were generally larger than the alterations used in Experiment 3. It is very likely indeed that our subjects more easily discriminated between standards and IT series having relatively large alterations than between standards and IT series having relatively small alterations. It is interesting to note that Cuddy et al. (1981, Experiment 2) also report that nondiatonic alterations were harder to detect in far-key transpositions than in near-key transpositions. Unfortunately, these authors provide no data for diatonic alterations.

The present set of experiments, using repeated standards and silent short-term RIs, show that tonal strength is of major importance in recognition. Dowling (1991), using novel tone series and both filled (long-term) and unfilled (short-term) intervals, concluded that tonal strength is of importance for long-term intervals only. In trying to relate these findings to those of the present experiments, it could be suggested that although tonal strength becomes a useful factor in recognition after a few repetitions of the standard, it is not useful for novel tone series. In this way, the number of repetitions of the standard might be (a part of) the explanation, but further research is needed before conclusions can be drawn. 


\section{REFERENCES}

Banks, W. P. (1970). Signal detection theory and human memory. Psychological Bulletin, 74, 81-99.

BEAL, A. L. (1985). The skill of recognizing musical structures. Memory \& Cognition, 13, 405-412.

Boltz, M., \& Jones, M. R. (1986). Does rule recursion make melodies easier to reproduce? If not, what does? Cognitive Psychology, $18,389-431$.

Brown, H., \& ButLer, D. (1981). Diatonic trichords as minimal tonal cue cells. In Theory Only, 5, 39-55.

Croonen, W. L. M., \& Kop, P. F. M. (1989). Tonality, tonal scheme and contour in delayed recognition of tone sequences. Music Perception, 7, 49-68.

Cuddy, L. L., \& CoHEN, A. J. (1976). Recognition of transposed melodic sequences. Quarterly Journal of Experimental Psychology, 28, 255-270.

Cuddy, L. L., Cohen, A. [J.], \& Mewhort, D. J. (1981). Perception of structure in short melodic sequences. Journal of Experimental Psychology: Human Perception \& Performance, 7, 869-883.

CudDY, L. L., \& Lyons, H. I. (1981). Musical pattern recognition: A comparison of listening to and studying tonal structures and tonal ambiguities. Psychomusicology, 1, 15-33.

DEuTsCH, D. (1970). Tones and numbers: Specificity of interference in short-term memory. Science, 68, 1604-1605.

DEUTSCH, D. (1980). The processing of structured and unstructured tonal sequences. Perception \& Psychophysics, 28, 381-389.

DeutsCh, D., \& FEROE, J. (1981). The internal representation of pitch series in tonal music. Psychological Review, 88, 503-522.

Dewar, K. M., Cuddy, L. L., \& MewhorT, D. J. (1977). Recognition memory for single tones with and without context. Journal of Experimental Psychology: Human Learning \& Memory, 3, 60-67.

DEWITT, L., \& Crowder, R. (1986). Recognition of novel melodies after brief delays. Music Perception, 3, 259-274.

Dowling, W. J. (1978). Scale and contour: Two components of a theory of memory for melodies. Psychological Review, 85, 341-354.

Dowling, W. J. (1991). Tonal strength and melody recognition after long and short delays. Perception \& Psychophysics, 50, 305-313.

Dowling, W. J., \& Fujitani, D. S. (1971). Contour, interval, and pitch recognition in memory for melodies. Journal of the Acoustical Society of America, 49, 524-531.

Dowling, W. J., \& Harwood, D. (1986). Music cognition. Orlando, FL: Academic Press.

Francès, R. (1958). La perception de la musique. Paris: J. Vrin.

GrEeN, D. M., \& SWETS, J. A. (1966). Signal detection theory and psychophysics. New York: Krieger.

Kaplan, H. L., Macmillan, N. A., \& Creelman, C. D. (1978). Tables of $d^{\prime}$ for variable-standard discrimination paradigms. Behavior Research Methods \& Instrumentation, 10, 796-813.

Krumhansl, C., Bharucha, J., \& Kessler, E. (1982). Perceived harmonic structure of chords in three related musical keys. Journal of
Experimental Psychology: Human Perception \& Performance, 8, 24-36.

Macmillan, N., Kaplan, H., \& Creelman, D. (1977). The psychophysics of categorical perception. Psychological Review, 84, 452-471.

MCNicoL, D. (1971). A primer of signal detection theory. London: Allen \& Unwin.

Norman, D. A. (1964). A comparison of data obtained with different false-alarm rates. Psychological Review, 71, 243-246.

Norman, D. A., \& Wickelgren, W. A. (1965). Short-term recognition memory for single digits and pairs of digits. Joumal of Experimental Psychology, 70, 479-489.

Pollack, I., \& Norman, D. A. (1964). A non-parametric analysis of recognition experiments. Psychonomic Science, 1, 125-126.

Pollack, I., Norman, D. A., \& Galanter, E. (1964). An efficient non-parametric analysis of recognition memory. Psychonomic Science, 1, 327-328.

SCHEFFE, H. A. (1953). A method for judging all possible contrasts in the analysis of variance. Biometrika, 40, 87-104.

SHEPARD, R. N., \& TEGHTSOONIAN, M. (1961). Retention of information under conditions approaching a steady state. Journal of Experimental Psychology, 62, 302-309.

SwETs, J. A. (1973). The relative operating characteristic in psychology. Science, 182, 990-1000.

WATKINS, A. J. (1985). Scale, key, and contour in the discrimination of tuned and mistuned approximations to melody. Perception \& Psychophysics, 37, 275-285.

\section{NOTES}

1. For series of both 7 and 10 tones, contour complexity was defined by the number of contour changes, where a strong contour was defined as having few contour changes. The number of contour changes for series with simple contours and a strong tonal structure was chosen in accordance with the number of chords that made up the series, in such a way that the directional changes would "separate" the chords as they were to be located at the "borders" of the chords. The 7-tone series consisted of three chords $\left(I-v i i^{0}-I\right)$, and so had two contour changes; the 10-tone series had four chords $(I-I V-V-I)$, and so had three changes. The complex contours were compiled by adding two changes to the number of changes of the simple contour.

2. This finding is possibly related to those of Cuddy and Cohen (1976), who found that different orders of the major triad also affect ease of recognition.

3. In the present experiment, series with a strong and a weak tonal structure were equally repeated, so an interpretation in terms of differential repetition of SS series versus WS series can be excluded. The present results strongly suggest that degree of underlying SS is a major factor in recognizing tone series.

(Manuscript received June 2, 1992 ; revision accepted for publication October 16, 1993.) 